\title{
COMPARING THE HAUSDORFF AND PACKING MEASURES OF SETS OF SMALL DIMENSION IN METRIC SPACES
}

\author{
TAPIO RAJALA
}

\begin{abstract}
We give a new estimate for the ratio of $s$-dimensional Hausdorff measure $\mathcal{H}^{s}$ and (radius-based) packing measure $\mathcal{P}^{s}$ of a set in any metric space. This estimate is

$$
\inf _{E} \frac{\mathcal{P}^{s}(E)}{\mathcal{H}^{s}(E)} \geq 1+\left(2-\frac{3}{2^{1 / s}}\right)^{s}
$$

where $0<s<1 / 2$ and the infimum is taken over all metric spaces $X$ and sets $E \subset X$ with $0<\mathcal{H}^{s}(E)<\infty$. As an immediate consequence we improve the upper bound for the lower $s$-density of such sets in $\mathbb{R}^{n}$.
\end{abstract}

\section{INTRODUCTION}

This paper investigates the dependence between Hausdorff and packing measures. Let us first recall how they are defined and fix some notation. Let $X$ be a metric space with a metric $d$. We denote an open ball by $B(x, r)=\{y \in X: d(x, y)<r\}$. A $\delta$-packing of a set $E \subset X$ is a countable collection of disjoint balls of radii at most $\delta$ and with centers in $E$. With $s \geq 0$ we let

$$
P_{\delta}^{s}(E)=\sup \left\{\sum_{B(x, r) \in \mathcal{B}}(2 r)^{s}: \mathcal{B} \text { is a } \delta \text {-packing of } E\right\} .
$$

From this the $s$-dimensional (radius-based) packing premeasure is defined to be

$$
P_{0}^{s}(E)=\lim _{\delta \downarrow 0} P_{\delta}^{s}(E) .
$$

Finally the s-dimensional (radius-based) packing measure of $E$ is defined as

$$
\mathcal{P}^{s}(E)=\inf \left\{\sum_{i=1}^{\infty} P_{0}^{s}\left(E_{i}\right): E \subset \bigcup_{i=1}^{\infty} E_{i}\right\} \text {. }
$$

2000 Mathematics Subject Classification. Primary 28A78.

Key words and phrases. Hausdorff measure and packing measure and density and metric space.

The author acknowledges the support of the Academy of Finland, project No. 211229. 
Given a set $E \subset X$ and $s>0$ the $s$-dimensional Hausdorff measure of the set $E$ is defined as

$$
\mathcal{H}^{s}(E)=\lim _{\delta \downarrow 0} \mathcal{H}_{\delta}^{s}(E)
$$

where

$$
\mathcal{H}_{\delta}^{s}(E)=\inf \left\{\sum_{i=1}^{\infty}\left|E_{i}\right|^{s}: E \subset \bigcup_{i=1}^{\infty} E_{i} \text { and }\left|E_{i}\right| \leq \delta\right\}
$$

and $|E|$ denotes the diameter of $E$. For more information on these measures, see for example [11].

There is also a diameter-based definition for the packing measure, where in the definition we replace $2 r$ by $|B(x, r)|$. In any normed space the diameter of a ball $B(x, r)$ is exactly $2 r$, and so the radius-based and diameterbased packing measures coincide. However, in general metric spaces these two definitions do not always agree. The proofs in this paper only work for the radius-based packing measure. Note that the radius-based packing measure has many nice properties in metric spaces which the diameterbased packing measure is lacking (see $[7,8,4,9]$ ).

We would like to understand how the Hausdorff and packing measures relate to each other. From $[13,14]$ we know that we always have $\mathcal{H}^{s}(E) \leq$ $\mathcal{P}^{s}(E)$ in Euclidean spaces. For almost every dimension this inequality is also strict. In fact, if it happens that $0<\mathcal{H}^{s}(E)=\mathcal{P}^{s}(E)<\infty$, then the dimension $s$ must be an integer and $E$ has to be $s$-rectifiable. This fact gives rise to the natural question: how close to equality can we get if $s$ is not an integer? We will prove an estimate for this for small $s$. This estimate holds in any metric space.

In the other direction there is no hope to obtain any bounds as it is possible to construct sets with $\mathcal{H}^{s}(E)=0$ and $\mathcal{P}^{s}(E)=\infty$. With $s>0$ let us denote

$$
c(s, X)=\inf _{E} \frac{\mathcal{P}^{s}(E)}{\mathcal{H}^{s}(E)},
$$

where the infimum is taken over all sets $E \subset X$ with $0<\mathcal{H}^{s}(E)<\infty$. We prove the following.

Theorem 1.1. For any metric space $X$ and $0<s<\frac{1}{2}$ we have

$$
c(s, X) \geq l_{2}(s)=1+\left(2-\frac{3}{2^{1 / s}}\right)^{s} .
$$

The previous non-trivial estimates for $c(s, \mathbb{R})$ are due to D.-J. Feng. He proved in [5] that

$$
c(s, \mathbb{R}) \geq l_{1}(s)=2^{s}\left(1+\min \left\{16^{-(1-s)^{-1}}, 8^{-(1-s)^{-2}}\right\}\right)^{s}
$$




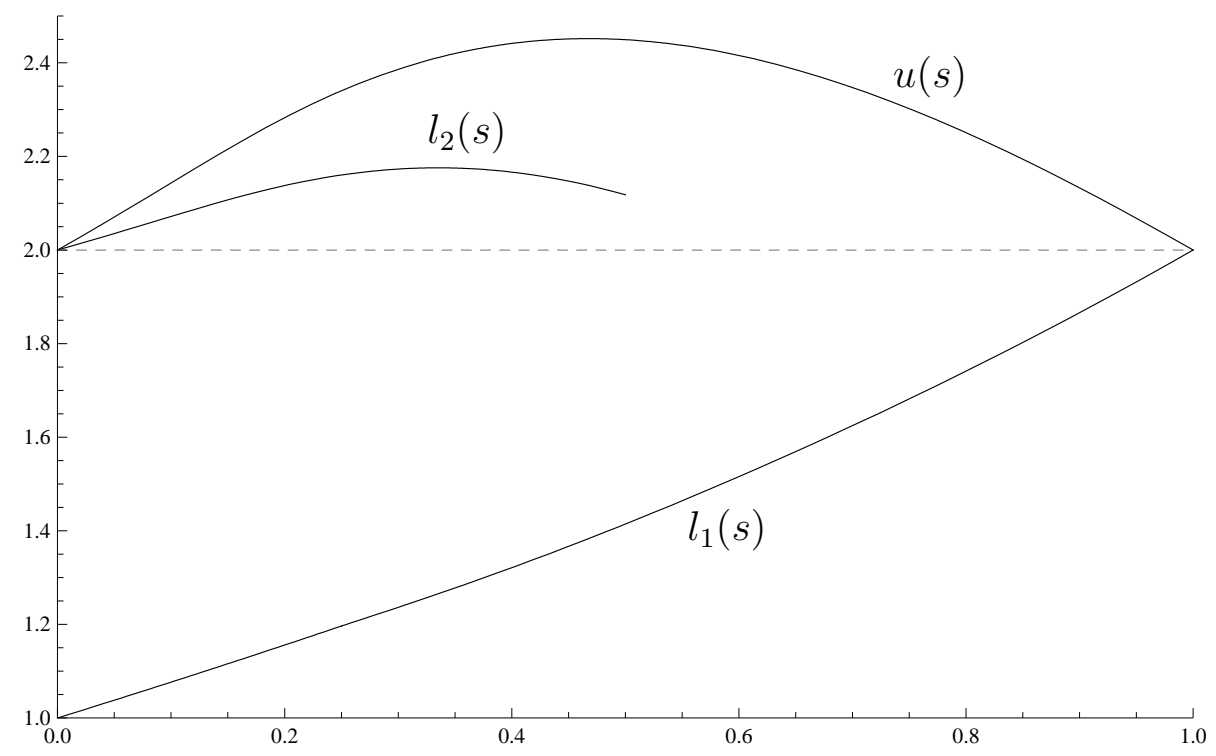

Figure 1. Here are the graphs of the bounds for $c(s, \mathbb{R})$. The upper-bound $u(s)$ and lower-bound $l_{1}(s)$ are due to Feng. In this paper we prove the lower-bound $l_{2}(s)$.

for $0<s<1$ and using his exact calculations for the packing measure of Cantor sets [6] he also gave the upper bound

$$
c\left(s, \mathbb{R}^{n}\right) \leq u(s)=2^{s}\left(2^{1 / s}-1\right)^{s}
$$

for $0<s<1$.

Our Theorem 1.1 improves the lower bound significantly for $0<s<1 / 2$. In addition, it holds in every metric space. See Figure 1 for an illustration of the bounds. Our proof for the new lower bound in metric spaces works only for dimensions $s$ with $0<s<1 / 2$. However, with similar methods the lower bound could be improved for $1 / 2 \leq s<1$ as well. As the exact value for $c(s, X)$ still remains open even for $0<s<1 / 2$, we will not pursue the possible improvements for the higher dimensions.

The Cantor sets on the real line which give the upper bound for $c\left(s, \mathbb{R}^{n}\right)$ can not be constructed in general metric spaces $X$. In fact, we have the following result concerning the upper bound.

Proposition 1.2. For every $0<s<1$ and every $c>0$ there exists a metric space $X$ with $c(s, X)>c$.

Proof. Take an integer $k \geq 2$. Define the space $X$ to be a Cantor set given as the limit of the iterated function system $\left\{f_{i}\right\}_{i=1}^{k}$ with

$$
f_{i}(x)=k^{-1 / s} x+\frac{i-1}{k} .
$$


This means that $X$ is the unique compact set in $\mathbb{R}$ with

$$
X=\bigcup_{i=1}^{k} f_{i}(X) .
$$

Now take any $E \subset X$ with $\mathcal{H}^{s}(E)>0$. Let $\delta>0$ so that $\mathcal{H}_{\delta}^{s}(E)>$ $\mathcal{H}^{s}(E) / 2$. For any $m \in \mathbb{N}$ so large that $k^{-(m-1) / s}<\delta$ the set $E$ must meet at least $\mathcal{H}_{\delta}^{s}(E) k^{m}$ construction pieces of the form $f_{i_{1}} \circ \cdots \circ f_{i_{1}}([0,1])$. On the other hand, each two of these construction pieces have distance $k^{-(m-1) / s}-k^{-m / s}$ between them. So,

$$
P_{\delta}^{s}(E) \geq \mathcal{H}_{\delta}^{s}(E) k^{m}\left(k^{-(m-1) / s}-k^{-m / s}\right)^{s} \geq k 2^{-s-1} \mathcal{H}^{s}(E) .
$$

Hence the ratio $P^{s}(E) / \mathcal{H}^{s}(E)$ can be made as large as we wish by choosing a large enough $k$.

Feng asked in [5] if it is true that $c(s, \mathbb{R})=u(s)$. Although we are not yet able to answer this question, we believe that this should be the case, see Remark 2.3.

Similarly as Feng proved a lower density theorem for Hausdorff measures from his estimate, we can prove the following corollary to Theorem 1.1 for sets in $\mathbb{R}^{n}$. See [5] for the proof.

Corollary 1.3. Let $0<s<\frac{1}{2}$ and $F \subset \mathbb{R}^{n}$ a Borel set with $\mathcal{H}^{s}(F)<\infty$. Then

$$
\liminf _{r \downarrow 0} \frac{\mathcal{H}^{s}(F \cap B(x, r))}{(2 r)^{s}} \leq \frac{1}{l_{2}(s)}<\frac{1}{2}
$$

for $\mathcal{H}^{s}$-almost all $x \in F$.

The first estimate for the lower density of Hausdorff measures on the real line was already proved by A. S. Besicovitch in [2]. He proved that for a set $E \subset \mathbb{R}$, with $0<\mathcal{H}^{s}(E)<\infty$, at $\mathcal{H}^{s}$-almost every point $x \in E$ we have

$$
\frac{2}{\left(2^{1+1 / s}-2\right)^{s}} \leq \limsup _{r \downarrow 0} \frac{\mathcal{H}^{s}(E \cap B(x, r))}{(2 r)^{s}} \leq 1
$$

and

$$
\liminf _{r \downarrow 0} \frac{\mathcal{H}^{s}(F \cap B(x, r))}{(2 r)^{s}} \leq 2^{-s} \limsup _{r \downarrow 0} \frac{\mathcal{H}^{s}(F \cap B(x, r))}{(2 r)^{s}} .
$$

Combining these two gives

$$
\liminf _{r \downarrow 0} \frac{\mathcal{H}^{s}(F \cap B(x, r))}{(2 r)^{s}} \leq 2^{-s} .
$$

More general estimates on the real line have been obtained in [10].

For an integer $k$ it is clear that $c\left(k, \mathbb{R}^{n}\right)=1$. In this case a more interesting question is the value of the constant when the infimum is taken over 
all purely $k$-unrectifiable sets. (See [11] for the definition and characterizations of rectifiability.) On the plane this question is the famous Besicovitch 1/2-problem [1,3]: If a set $E \subset \mathbb{R}^{2}$ with $0<\mathcal{H}^{1}(E)<\infty$ has

$$
\liminf _{r \downarrow 0} \frac{\mathcal{H}^{1}(F \cap B(x, r))}{2 r}>\frac{1}{2}
$$

at $\mathcal{H}^{1}$-almost every point $x \in E$, is it then 1 -rectifiable? Currently the best known result is by D. Preiss and J. Tišer [12]. It says that this is the case when we replace $1 / 2$ by $(2+\sqrt{46}) / 12$.

\section{Proof of the theOrem}

We prove Theorem 1.1 with the help of the following two geometric lemmas. The first lemma tells us that most of the balls in a nearly optimal packing must have certain empty annular regions.

Lemma 2.1. Let $0<s<\frac{1}{2}$. There exists a constant $0<\epsilon_{s}<\infty$ depending only on $s$ so that for every set $E \subset X$, radius $r>0$ and point $x \in E$, and for every $0<\epsilon<\epsilon_{s}$ the following holds. If

$$
E \cap(B(x, r-\epsilon r) \backslash B(x, r / 2)) \neq \emptyset
$$

then there exist two disjoint balls $B\left(x_{1}, r_{1}\right), B\left(x_{2}, r_{2}\right) \subset B(x, r)$ so that $x_{1}, x_{2} \in E$ and

$$
\left(2 r_{1}\right)^{s}+\left(2 r_{2}\right)^{s}>(1+\epsilon)(2 r)^{s}
$$

Proof. Without loss of generality we may assume that $r=1$. Assume first that there is a point $y \in E \cap(B(x, 1-\epsilon) \backslash B(x, 2 / 3))$. Denote $t=1-|x-y|$. Now

and

$$
B(x, 1-2 t) \cap B(y, t)=\emptyset
$$

$$
B(x, 1-2 t), B(y, t) \subset B(x, 1) .
$$

Write $f_{s}(t)=(2-4 t)^{s}+(2 t)^{s}$. Observe that

$$
f_{s}^{\prime \prime}(t)=4 s(s-1)\left((2 t)^{s-2}+4(2-4 t)^{s-2}\right)<0
$$

for every $t \in[0,1 / 3]$ and hence $f_{s}$ is concave on that interval. Next notice that $f_{s}(0)=2^{s}$ and $f_{s}(1 / 3)=2(2 / 3)^{s}$. Therefore because $\lim _{t \downarrow 0} f_{s}^{\prime}(t)=\infty$ there exists $\epsilon_{1}>0$ so that $f_{s}(t)-f_{s}(0)>2 t$ for every $0<t<\epsilon_{1}$.

Next we assume that there is a point $z \in E \cap(B(x, 2 / 3) \backslash B(x, 1 / 2))$. Now let $h=|x-z|$. Clearly

$$
B(x, h / 2) \cap B(z, h / 2)=\emptyset
$$

and

Furthermore,

$$
B(x, h / 2), B(z, h / 2) \subset B(x, 1) .
$$

$$
2(h)^{s} \geq 2^{1-s}>(1+\epsilon) 2^{s}
$$


when $\epsilon<2^{1-2 s}-1$. Taking $\epsilon_{s}=\min \left\{\epsilon_{1}, 2^{1-2 s}-1\right\}$ finishes the proof.

In our second lemma we show that if the Hausdorff content inside a ball $B(x, r)$ is large enough, then there exists a large $\delta$-packing inside the ball $B(x, 2 r)$. The idea is to first find three points in the ball which are far enough from each other. Then around the two points, who have the shortest distance between them, we find balls with radius equal to half the distance. The last point will be given the largest possible ball around it.

Lemma 2.2. Let $\delta>0$. Suppose we are given an exponent $s \in(0,1 / 2)$, a set $E \subset X$, a radius $r \in(0, \delta / 2)$ and a point $x \in E$. If $\mathcal{H}_{\delta}^{s}(E \cap B(x, r))>c$ then there exists a packing $\left\{B\left(x_{1}, r_{1}\right), B\left(x_{2}, r_{2}\right), B\left(x_{3}, r_{3}\right)\right\}$ of $E \cap B(x, r)$ inside the ball $B(x, 2 r)$ so that

$$
\left(2 r_{1}\right)^{s}+\left(2 r_{2}\right)^{s}+\left(2 r_{3}\right)^{s} \geq\left(1+\left(2-\frac{3}{2^{1 / s}}\right)^{s}\right) c .
$$

Proof. Our aim is to find the centers $x_{1}, x_{2}, x_{3} \in E \cap B(x, r)$ which have the following properties: the distances between the points are in the order

$$
d\left(x_{1}, x_{2}\right) \geq d\left(x_{1}, x_{3}\right) \geq d\left(x_{2}, x_{3}\right)>\left(\frac{c}{2}\right)^{1 / s}
$$

and either

$$
d\left(x_{1}, x_{2}\right)>c^{1 / s}
$$

or

$$
d\left(x_{1}, x_{3}\right)>c^{1 / s}-\left(\frac{c}{2}\right)^{1 / s}
$$

Let us now find these centers. Because $\mathcal{H}_{\delta}^{s}(E \cap B(x, r))>c$ there exist points $y_{1}, y_{2} \in B(x, r) \cap E$ so that $d\left(y_{1}, y_{2}\right)>c^{1 / s}$. Assume first that

$$
\min \left\{d\left(y_{1}, x\right), d\left(y_{2}, x\right)\right\}>(c / 2)^{1 / s} .
$$

Now selecting the points $x_{1}, x_{2}$ and $x_{3}$ among the points $x, y_{1}$ and $y_{2}$ so that (2) holds is enough, since then automatically inequality (3) holds.

In the case the inequality (5) is not true we have to choose the points $x_{1}, x_{2}$ and $x_{3}$ differently. We may assume that

$$
\mathcal{H}_{\delta}^{s}\left(\left\{z \in E \cap B(x, r): d\left(z, y_{1}\right) \leq d\left(z, y_{2}\right)\right\}\right)>\frac{c}{2}
$$

is true. Then there are points $z_{1}, z_{2} \in\left\{z \in E \cap B(x, r): d\left(z, y_{1}\right) \leq d\left(z, y_{2}\right)\right\}$ so that $d\left(z_{1}, z_{2}\right)>(c / 2)^{1 / s}$. By changing $z_{1}$ or $z_{2}$ to be $y_{1}$ if necessary, we can assume that either $z_{1}=y_{1}$ or $\max \left\{d\left(y_{1}, z_{1}\right), d\left(y_{1}, z_{2}\right)\right\} \leq(c / 2)^{1 / s}$. Now choose the points $x_{1}, x_{2}$ and $x_{3}$ from the points $y_{2}, z_{1}$ and $z_{2}$ so that (2) holds. If it happens that $z_{1}=y_{1}$ then (3) is true. If not then (4) holds.

Now that the centers of our balls have been found, we define the radii to be $r_{2}=r_{3}=d\left(x_{2}, x_{3}\right) / 2$ and $r_{1}=\max \left\{c^{1 / s}-3 r_{2}, r_{2}\right\}$. Because of conditions (2), (3) and (4) the balls $B\left(x_{i}, r_{i}\right)$ are pairwise disjoint. 
Let us check next that $B\left(x_{i}, r_{i}\right) \subset B(x, 2 r)$ for each $i$. Since $r_{2}=r_{3} \leq r$, this clearly holds for $i=2,3$ and for $i=1$ when $r_{1}=r_{2}$. If (5) is true, then $x_{1}, x_{2}$ and $x_{3}$ are chosen from the points $x, y_{1}$ and $y_{2}$. Therefore one of them is $x$. As $x_{1} \in B(x, r)$, we must then have $r_{1}<r$. (If this were not the case, then the balls would not be disjoint.) Thus $B\left(x_{1}, r_{1}\right) \subset B(x, 2 r)$.

The only case left to check is when $r_{1}=c^{1 / s}-3 r_{2}$ and (5) does not hold. But then

$$
\begin{aligned}
r_{1}=c^{1 / s}-3 r_{2} & <d\left(y_{1}, y_{2}\right)-3 r_{2} \leq d\left(y_{1}, x\right)+d\left(y_{2}, x\right)-3 r_{2} \\
& \leq r+\left(\frac{c}{2}\right)^{1 / s}-\frac{3}{2}\left(\frac{c}{2}\right)^{1 / s}<r
\end{aligned}
$$

Next we confirm (1). Let us do this first in the case $r_{1}=r_{2}$. Then $r_{2} \geq c^{1 / s}-3 r_{2}$ from which we get $r_{2} \geq \frac{c^{1 / s}}{4}$ and consequently

$$
\left(2 r_{1}\right)^{s}+\left(2 r_{2}\right)^{s}+\left(2 r_{3}\right)^{s} \geq 3\left(\frac{c^{1 / s}}{2}\right)^{s} \geq\left(1+\left(2-\frac{3}{2^{1 / s}}\right)^{s}\right) c .
$$

Now we deal with the case $r_{1}=c^{1 / s}-3 r_{2}$. From the inequalities (2) and $r_{2} \leq \frac{c^{1 / s}}{4}$ we get $2^{-1 / s} \leq \frac{d\left(x_{2}, x_{3}\right)}{c^{1 / s}} \leq 2^{-1}$. Finally we confirm (1) by calculating

$$
\begin{aligned}
\left(2 r_{1}\right)^{s}+\left(2 r_{2}\right)^{s}+\left(2 r_{3}\right)^{s} & =2 d\left(x_{2}, x_{3}\right)^{s}+\left(2 c^{1 / s}-3 d\left(x_{2}, x_{3}\right)\right)^{s} \\
& =\left(2 \frac{d\left(x_{2}, x_{3}\right)^{s}}{c}+\left(2-3 \frac{d\left(x_{2}, x_{3}\right)}{c^{1 / s}}\right)^{s}\right) c \\
& \geq\left(1+\left(2-\frac{3}{2^{1 / s}}\right)^{s}\right) c
\end{aligned}
$$

where the last inequality follows by investigating the function

$$
f_{s}(t)=\left(2 t^{s}+(2-3 t)^{s}\right) c .
$$

Simply by noticing that $f_{s}^{\prime \prime}(t)<0$ for every $2^{-1 / s}<t<2^{-1}$ and $f_{s}\left(2^{-1 / s}\right) \leq$ $f_{s}\left(2^{-1}\right)$ we obtain $f_{s}(t) \geq f_{s}\left(2^{-1 / s}\right)$ for every $0<s<1 / 2$ and every $2^{-1 / s}<t<2^{-1}$.

We are now ready to prove Theorem 1.1. The strategy is to take a $\delta$ packing which is supposed to be nearly optimal. If the Hausdorff measure is large inside a ball which is used in the packing, by the previous lemmas we are able to replace the ball with a slightly better packing. Because the $\delta$-packing was nearly optimal, this can not happen to too many balls.

of Theorem 1.1. We show that for every $E \subset X$ and $\delta>0$

$$
l_{2}(s) \mathcal{H}_{\delta}^{s}(E) \leq P_{\delta}^{s}(E) .
$$

We may assume that $P_{\delta}^{s}(E)<\infty$. Let $c$ and $\epsilon_{0}$ be the constants from Lemma 2.1. Take $0<\epsilon<\epsilon_{s}$ and let $\left\{B\left(x_{i}, r_{i}\right)\right\}, i \in I$, be a finite $\delta$-packing 
of $E$ so that

$$
\sum_{i \in I}\left(2 r_{i}\right)^{s} \geq P_{\delta}^{s}(E)-\epsilon^{2}
$$

First we estimate

$$
\mathcal{H}_{\delta}^{s}\left(E \backslash \bigcup_{i \in I} B\left(x_{i},(1-\epsilon) r_{i}\right)\right)
$$

from above. To do this we define for each

$$
x \in E \backslash \bigcup_{i \in I} B\left(x_{i},(1-\epsilon) r_{i}\right)
$$

a radius

$$
r_{x}=\min \left\{\delta / 5, \operatorname{dist}\left(\{x\}, \bigcup_{i \in I} B\left(x_{i},(1-2 \epsilon) r_{i}\right)\right)\right\},
$$

where $\operatorname{dist}(\cdot, \cdot)$ denotes the distance between the sets. Then using $5 r$ covering theorem we find a collection $\left\{y_{j}\right\}_{j \in J}$ of points so that the balls $\left\{B\left(y_{j}, r_{y_{j}}\right)\right\}_{j \in J}$ are pairwise disjoint and

$$
E \backslash \bigcup_{i \in I} B\left(x_{i},(1-\epsilon) r_{i}\right) \subset \bigcup_{j \in J} B\left(y_{j}, 5 r_{y_{j}}\right) .
$$

From $P_{\delta}^{s}(E)<\infty$ it follows that $\left\{y_{j}\right\}_{j \in J}$ must be countable. Then

$$
\begin{aligned}
\mathcal{H}_{\delta}^{s}\left(E \backslash \bigcup_{i \in I} B\left(x_{i},(1-\epsilon) r_{i}\right)\right) & \leq 5^{s} \sum_{j \in J}\left(2 r_{y_{j}}\right)^{s} \leq 5^{s}\left(P_{\delta}^{s}(E)-\sum_{i \in I}\left(2(1-2 \epsilon) r_{i}\right)^{s}\right) \\
& \leq 5^{s}\left(\epsilon^{2}+\left(1-(1-2 \epsilon)^{s}\right) P_{\delta}^{s}(E)\right) .
\end{aligned}
$$

Now divide $I$ into three sets,

$$
\begin{aligned}
& I_{1}=\left\{i \in I: \mathcal{H}_{\delta}^{s}\left(E \cap B\left(x_{i},(1-\epsilon) r_{i}\right)\right) \leq\left(l_{2}(s)^{-1}+\epsilon\right)\left(2 r_{i}\right)^{s}\right\}, \\
& I_{2}=\left\{i \in I \backslash I_{1}: E \cap\left(B\left(x_{i},(1-\epsilon) r_{i}\right) \backslash B\left(x, r_{i} / 2\right)\right) \neq \emptyset\right\} \text { and } \\
& I_{3}=I \backslash\left(I_{1} \cup I_{2}\right) .
\end{aligned}
$$

For every $i \in I_{2}$ we can use Lemma 2.1 to obtain two disjoint balls $B\left(x_{i, 1}, r_{i, 1}\right), B\left(x_{i, 2}, r_{i, 2}\right) \subset B\left(x_{i}, r_{i}\right)$ so that $x_{i, 1}, x_{i, 2} \in E$ and

$$
\left(2 r_{i, 1}\right)^{s}+\left(2 r_{i, 2}\right)^{s}>(1+\epsilon)\left(2 r_{i}\right)^{s} \text {. }
$$

Let $i \in I_{3}$. Then $\mathcal{H}_{\delta}^{s}\left(E \cap B\left(x_{i}, r_{i} / 2\right)\right)>\left(l_{2}(s)^{-1}+\epsilon\right)\left(2 r_{i}\right)^{s}$. Lemma 2.2 then gives pairwise disjoint balls $B\left(x_{i, 1}, r_{i, 1}\right), B\left(x_{i, 2}, r_{i, 2}\right), B\left(x_{i, 3}, r_{i, 3}\right) \subset B\left(x_{i}, r_{i}\right)$ with $x_{i, 1}, x_{i, 2}, x_{i, 3} \in E$ and

$$
\left(2 r_{i, 1}\right)^{s}+\left(2 r_{i, 2}\right)^{s}+\left(2 r_{i, 3}\right)^{s} \geq l_{2}(s)\left(l_{2}(s)^{-1}+\epsilon\right)\left(2 r_{i}\right)^{s}>(1+\epsilon)\left(2 r_{i}\right)^{s} .
$$


Now

$$
\begin{aligned}
P_{\delta}^{s}(E) & \geq \sum_{i \in I_{1}}\left(2 r_{i}\right)^{s}+\sum_{i \in I_{2}} \sum_{k=1}^{2}\left(2 r_{i, k}\right)^{s}+\sum_{i \in I_{3}} \sum_{k=1}^{3}\left(2 r_{i, k}\right)^{s} \\
& >\sum_{i \in I_{1}}\left(2 r_{i}\right)^{s}+(1+\epsilon) \sum_{i \in I_{2} \cup I_{3}}\left(2 r_{i}\right)^{s} \geq P_{\delta}^{s}(E)-\epsilon^{2}+\epsilon \sum_{i \in I_{2} \cup I_{3}}\left(2 r_{i}\right)^{s}
\end{aligned}
$$

and so

$$
\sum_{i \in I_{2} \cup I_{3}}\left(2 r_{i}\right)^{s}<\epsilon
$$

We finish the proof by calculating

$$
\begin{aligned}
\mathcal{H}_{\delta}^{s}(E) & \leq \mathcal{H}_{\delta}^{s}\left(E \backslash \bigcup_{i \in I} B\left(x_{i},(1-\epsilon) r_{i}\right)\right)+\sum_{i \in I} \mathcal{H}_{\delta}^{s}\left(E \cap B\left(x_{i},(1-\epsilon) r_{i}\right)\right) \\
& \leq \mathcal{H}_{\delta}^{s}\left(E \backslash \bigcup_{i \in I} B\left(x_{i},(1-\epsilon) r_{i}\right)\right)+\sum_{i \in I_{1}}\left(l_{2}(s)^{-1}+\epsilon\right)\left(2 r_{i}\right)^{s}+\sum_{i \in I_{2} \cup I_{3}}\left(2 r_{i}\right)^{s} \\
& \leq 5^{s}\left(\epsilon^{2}+\left(1-(1-2 \epsilon)^{s}\right) P_{\delta}^{s}(E)\right)+\left(l_{2}(s)^{-1}+\epsilon\right) P_{\delta}^{s}(E)+\epsilon
\end{aligned}
$$

Remark 2.3. In Lemma 2.2 we could use two balls instead of three. This would give $l_{2}(s)=2$. The point of using three balls was to give an estimate which shows that $c(s, X)>2$.

The method of the Lemma 2.2 of dividing a set into two subsets and choosing the one with larger Hausdorff content could be probably pushed further by dividing infinitely many times and comparing possible packings to a packing of a Cantor set. However, as we disregard at each step the set with smaller Hausdorff content, this method should only be able to give a geometric sequence of balls and thus an estimate $c(s, X) \geq 2^{s+1}\left(\frac{2^{1 / s}-1}{2^{1 / s}+1}\right)^{s}$. This would still not solve the exact value of $c(s, \mathbb{R})$ even for small $s$. However, a more sophisticated treatment carried out using both parts of the set might be able to do so.

Acknowledgments. We thank De-Jun Feng for the valuable comments he made on an earlier version of this paper.

\section{REFERENCES}

[1] A. S. Besicovitch: On the fundamental geometrical properties of linearly measurable plane sets of points, Math. Ann. 98, 422-464 (1928).

[2] A. S. Besicovitch, On linear sets of points of fractional dimension, Math. Ann. 101, no. 1, 161-193 (1929).

[3] A. S. Besicovitch: On the fundamental geometrical properties of linearly measurable plane sets of points II, Math. Ann. 115, 296-329 (1938).

[4] C. D. Cutler, The density theorem and Hausdorff inequality for packing measure in general metric spaces, Illinois J. Math. 39, no. 4, 676-694 (1995). 
[5] D.-J. Feng: Comparing packing measures to Hausdorff measures on the line, Math. Nachr. 241, 65-72 (2002).

[6] D.-J. Feng: Exact packing measure of linear Cantor sets, Math. Nachr. 248/249, 102-109 (2003).

[7] H. Haase: Non- $\sigma$-finite sets for packing measure, Mathematika 33 (1986), no. 1, $129-136$.

[8] H. Joyce: A space on which diameter-type packing measure is not Borel regular, Proc. Amer. Math. Soc. 127, no. 4, 985-991 (1999).

[9] H. Joyce and D. Preiss: On the existence of subsets of finite positive packing measure, Mathematika 42, 15-24 (1995).

[10] P. Mattila: Densities of measures on the real line, Ann. Acad. Sci. Fenn. Ser. A I Math. 4, no. 1, 53-61 (1979).

[11] P. Mattila: Geometry of Sets and Measures in Euclidean Spaces: Fractals and Rectifiability, Cambridge Univ. Press, Cambridge, (1995).

[12] D. Preiss and J. Tišer: On Besicovitch's $\frac{1}{2}$-problem, J. London Math. Soc. (2) 45, no. 2, 279-287 (1992).

[13] X. Saint Raymond and C. Tricot: Packing regularity of sets in $n$-space, Math. Proc. Cambridge Philos. Soc. 103, no. 1, 133-145 (1988).

[14] S. Taylor and C. Tricot: Packing measure, and its evaluation for a Brownian path, Trans. Amer. Math. Soc. 288, no. 2, 679-699 (1985).

Department of Mathematics and Statistics, P.O. Box 35 (MaD), FI-40014

UNIVERSITY OF JYVÄSKYLÄ, FINLAND

E-mail address: tapio.m.rajala@jyu.fi 